\section{(1)}

CrossMark

\title{
Trilateral overlap of tuberculosis, diabetes and HIV-1 in a high-burden African setting: implications for TB control
}

\author{
Tolu Oni ${ }^{1,2}$, Natacha Berkowitz ${ }^{1,2}$, Mmamapudi Kubjane ${ }^{1}$, Rene Goliath ${ }^{2}$, \\ Naomi S. Levitt ${ }^{3,6}$ and Robert J. Wilkinson $2,4,5,6$
}

\begin{abstract}
Affiliations: ${ }^{1}$ Division of Public Health Medicine, School of Public Health and Family Medicine, University of Cape Town, Cape Town, South Africa. ${ }^{2}$ Wellcome Centre for Infectious Disease Research in Africa, Institute of Infectious Disease and Molecular Medicine, University of Cape Town, Cape Town, South Africa. ${ }^{3}$ Division of Diabetes and Endocrinology, Dept of Medicine, Groote Schuur Hospital and Chronic Disease Initiative for Africa, Cape Town, South Africa. ${ }^{4}$ The Francis Crick Institute, London, UK. ${ }^{5}$ Dept of Medicine, Imperial College London, London, UK. 'Joint senior authors.
\end{abstract}

Correspondence: Tolu Oni, Room 4.41, Falmouth Building, Faculty of Health Sciences, University of Cape Town, Anzio Road, Observatory, Cape Town 7925, South Africa. E-mail: tolullah.oniduct.ac.za

@ERSpublications

DM/TB association is significant in HIV infection, but more accurate glycaemia markers at TB diagnosis are needed http://ow.ly/ZXoS30aPz22

Cite this article as: Oni T, Berkowitz N, Kubjane M, et al. Trilateral overlap of tuberculosis, diabetes and HIV-1 in a high-burden African setting: implications for TB control. Eur Respir J 2017; 50: 1700004 [https://doi.org/10.1183/13993003.00004-2017].

ABSTRACT The diabetes mellitus burden is growing in countries where tuberculosis (TB) and HIV-1 remain major challenges, threatening $\mathrm{TB}$ control efforts. This study determined the association between TB and diabetes/impaired glucose regulation in the context of HIV-1.

A cross-sectional study was conducted at a TB clinic in Cape Town (South Africa). Participants were screened for diabetes and impaired glucose regulation using fasting plasma glucose, oral glucose tolerance test and glycated haemoglobin (HbAlc).

$414 \mathrm{~TB}$ and 438 non-TB participants were enrolled. In multivariable analysis, diabetes was associated with TB (OR 2.4, 95\% CI 1.3-4.3; p=0.005), with 14\% population-attributable risk fraction; however, this association varied by diagnostic test (driven by HbAlc). The association remained significant in HIV-1-infected individuals (OR 2.4, 95\% CI 1.1-5.2; $\mathrm{p}=0.030)$. A high prevalence of impaired glucose regulation $(65.2 \%$ among TB cases) and a significant association with TB (OR 2.3, 95\% CI 1.6-3.3; $\mathrm{p}<0.001)$ was also found.

Diabetes and impaired glucose regulation prevalence was high and associated with TB, particularly in HIV-1infected individuals, highlighting the importance of diabetes screening. The variation in findings by diagnostic test highlights the need for better glycaemia markers to inform screening in the context of TB and HIV-1.

Received: Jan 032017 | Accepted after revision: April 082017

Earn CME accreditation by answering questions about this article. You will find these at erj.ersjournals.com/journal/cme

Support statement: This study was funded by the Clinical Infectious Disease Research Initiative, as part of a Wellcome Trust Strategic Grant (WT 084323, 104873), a Carnegie Corporation Postdoctoral Fellowship and a Harry Crossley Senior Clinical Fellowship. The Francis Crick Institute received support from Cancer Research UK, Research Councils UK and the Wellcome Trust. R.J. Wilkinson receives support from the National Research Foundation of South Africa (96841). The funders played no role in the design, conduct or analysis of the study. Funding information for this article has been deposited with the Crossref Funder Registry.

Conflicts of interest: None declared.

Copyright CERS 2017. This ERJ Open article is open access and distributed under the terms of the Creative Commons Attribution Licence 4.0. 


\section{Introduction}

Tuberculosis (TB) remains a health challenge in most low- and middle-income countries, including those in sub-Saharan Africa. Poverty, smoking, alcoholism, HIV-1 and diabetes mellitus are known drivers of the TB epidemic [1]. Diabetes triples the risk of TB and is associated with adverse outcomes [2-4]. The $\mathrm{TB} /$ diabetes association is increasingly important in low- and middle-income countries experiencing a growing burden of noncommunicable disease. According to the 2013 Global Burden of Disease Study, HIV-1, TB and diabetes rank first, third and ninth among the top 10 causes of life-years lost in South Africa [5].

The prevalence of diabetes among TB patients varies by geographic region and background diabetes population in the general population, with a higher prevalence reported in South Asian (25.3-44\%) and Latin American (14-39\%) populations [6-12]. In sub-Saharan Africa, diabetes prevalence in TB patients ranges from $8.5 \%$ to $16.4 \%$, although a large proportion of diabetes in sub-Saharan Africa remains undiagnosed [13-17]. Of note, most studies have used fasting or random blood glucose to diagnose diabetes, which may underestimate the true prevalence. A study in South Africa estimated the general population prevalence of diabetes and impaired glucose tolerance to be $12.3 \%$ and $11.2 \%$, respectively [18].

The increased risk of TB associated with diabetes is well documented. However, this risk is variable dependent on the population studied. A systematic review that reported a 3-fold increased risk of TB in diabetes patients did not include studies from sub-Saharan Africa where there are competing TB risk factors such as HIV-1 [2]. Recent studies in African settings showed increased odds of TB among diabetes patients with the exception of one study [19], possibly due to the low background prevalence of diabetes. A Ugandan study found HIV-1-infected TB patients to have reduced odds of diabetes compared with HIV-1-uninfected patients [20] and a Tanzanian study showed a stronger TB/diabetes association observed among HIV-1-uninfected (OR 4.23, 95\% CI 1.54-11.57) compared with HIV-1-infected (OR 0.14, 95\% CI 0.01-1.81) individuals [21]. The reasons for this apparent incongruity in the associations between TB, diabetes and HIV-1 are unclear as there are only a limited number of published studies [22].

The only South African study that assessed the prevalence of diabetes, using the oral glucose tolerance test (OGTT), among TB patients was conducted in 1980, when South Africa was $42 \%$ urbanised (compared with $62 \%$ urbanised today), and reported a $2.1 \%$ prevalence [16]. South Africa has the largest antiretroviral therapy (ART) programme globally and is ranked as having the sixth highest TB incidence globally [23, 24]. Given this HIV-1/TB burden, reducing the TB burden in South Africa is a priority in the global fight against TB. The strong association between HIV-1 and TB, dysglycaemic effects of some ART drugs, especially protease inhibitors and nonnucleoside reverse transcriptase inhibitors $[25,26]$, and the emerging diabetes epidemic highlight the importance of investigating the association between $\mathrm{TB}$, diabetes and HIV-1 in this setting, the results of which will have implications for TB control strategies. This study investigated the prevalence of diabetes and impaired glucose regulation (IGR), the association between TB and diabetes/IGR, and the population-attributable risk of TB due to diabetes in South Africa.

\section{Methods}

\section{Study setting and population}

In South Africa, TB patients in the public sector are largely screened and treated in TB clinics. This study was conducted at the largest TB clinic in Khayelitsha, a peri-urban township of around 390000 predominantly black Africans, in Cape Town, Western Cape province. In this province, diabetes, HIV-1 and TB rank as the first, third and fourth leading causes of death, respectively [27]. The 2012 HIV-1 antenatal prevalence in Khayelitsha was 34\% (95\% CI 31.0-36.6\%) (Western Cape Dept of Health, Cape Town, South Africa; 2012 Antenatal Survey, unpublished data) and the 2015 TB case notification rate was 917 per 100000 population (V. de Azevedo, City Health Manager, Khayelitsha, Cape Town, South Africa; personal communication), with a $60 \% \mathrm{HIV}-1 / \mathrm{TB}$ co-infection rate [28].

\section{Study design and sampling}

We conducted a cross-sectional study on consecutive patients with respiratory symptoms presenting to the clinic from July 2013 from August 2015. Patients were eligible if they provided consent, were aged $\geqslant 18$ years and had not received $>48 \mathrm{~h}$ of $\mathrm{TB}$ chemotherapy. Those who were critically ill and in need of emergency clinical care were ineligible as they were too physically unwell to give informed consent. The study was approved by the University of Cape Town Human Research Ethics Committee (HREC 403/ 2011). Assuming a 3-fold higher diabetes prevalence in HIV-1-uninfected TB cases, $7 \%$ diabetes prevalence in HIV-1-uninfected individuals, $70 \%$ of TB cases HIV-1-co-infected and $80 \%$ power, the study aimed to recruit $400 \mathrm{~TB}$ and 400 non-TB participants. 


\section{Study procedures}

Case definitions

TB cases were diagnosed according to South Africa guidelines with the GeneXpert system (Cepheid, Sunnyvale, CA, USA) and analysed in a centralised national health laboratory [29]. Non-TB participants were those with a negative $\mathrm{TB}$ diagnosis and resolution of respiratory symptoms. All participants were encouraged to undertake an HIV-1 test and were tested for diabetes using all three of the following: fasting plasma glucose (FPG), a 2-h OGTT and glycated haemoglobin (HbA1c). Diabetes diagnosis was defined as self-reported diabetes, FPG $\geqslant 7.0 \mathrm{mmol} \cdot \mathrm{L}^{-1}$, OGTT $\geqslant 11.1 \mathrm{mmol} \cdot \mathrm{L}^{-1}$ or $\mathrm{HbA} 1 \mathrm{c} \geqslant 6.5 \%[30,31]$. IGR was defined as FPG $5.5-<7.0 \mathrm{mmol} \cdot \mathrm{L}^{-1}$, OGTT $7.7-<11.1 \mathrm{mmol} \cdot \mathrm{L}^{-1}$ or HbA1c $5.7-<6.5 \%$.

\section{Measurements}

Venous blood was drawn from the antecubital vein at 0 (after an overnight fast) and 120 min in evacuated fluoride (glucose) and EDTA (HbA1c) tubes. All blood samples were processed on the day of collection at a centralised national health laboratory using standardised operating procedures of the cobas c311 (Roche/ Hitachi, Basel, Switzerland) system analyser assay. Weight, height and waist circumference were measured using standardised techniques [32]. Body mass index (BMI) was categorised as underweight $<18.5 \mathrm{~kg} \cdot \mathrm{m}^{-2}$, normal $18.5-24.9 \mathrm{~kg} \cdot \mathrm{m}^{-2}$, overweight $25-29.9 \mathrm{~kg} \cdot \mathrm{m}^{-2}$ and obese $\geqslant 30 \mathrm{~kg} \cdot \mathrm{m}^{-2}$ [32]. The cut-point for high waist circumference was $\geqslant 94 \mathrm{~cm}$ for males and $\geqslant 88 \mathrm{~cm}$ for females [32]. Hypertension was defined as a single measured blood pressure of systolic blood pressure $>140 \mathrm{mmHg}$ or diastolic blood pressure $>90 \mathrm{mmHg}[31]$, or a pre-existing diagnosis.

\section{Questionnaire}

Chronic disease risk factors were ascertained using the STEPs instrument [33]. Information on socioeconomic, demographic and medical history was collected using a researcher-administered questionnaire.

\section{Statistical analysis}

Medians (interquartile ranges (IQRs)) and proportions were used to summarise continuous and categorical variables, respectively. Chi-squared and Fisher's exact tests assessed associations between categorical variables. The Mann-Whitney test was used to compare medians between two groups and the Kruskal-Wallis test for more than two groups. A multivariable logistic regression model for the association between $\mathrm{TB}$ and diabetes was built manually, using forward selection, controlling for potential confounding variables. In order to retain statistical power and reduce potential biases, in the regression analysis involving the key variable HIV serostatus, multiple imputation was used to impute HIV serostatus for 50 participants with unknown HIV-1 status. Imputation using the chained equations method was implemented in Stata (StataCorp, College Station, TX, USA) using the "ice" command. Five imputed datasets were created. The "mi" Stata command was used to perform logistic regression analysis on the combined imputed datasets. The base model included a priori confounding variables of age and sex. Variables associated with TB $(\mathrm{p}<0.10)$ on univariable analysis were then added, sequentially including variables that improved the model based on the significant lowering of the Akaike Information Criterion in nonnested model comparisons. Potential effect modification between variables was determined by exploring the statistical significance of interaction variables. The fit of the model was assessed using Pearson's goodness-of-fit test with $\mathrm{p}>0.05$ indicating a good fit. The population-attributable risk was calculated as: $A R$ popdiabetes $=\mathrm{p}($ diabetes $)(\mathrm{OR}-1) /(1+\mathrm{p}($ diabetes $)(\mathrm{OR}-1))$, where $\mathrm{p}($ diabetes $)$ is general population diabetes prevalence and OR is the adjusted odds ratio [34]. Statistical significance was set at $\mathrm{p}<0.05$. All data were analysed using Stata version 13.0.

\section{Results}

\section{Baseline characteristics}

986 participants were recruited. 48 participants (4.9\%) were excluded as they had an undetermined TB status (TB could not be confirmed or excluded). A further 86 participants did not complete diabetes screening at baseline (did not return for fasting bloods). 852 participants were therefore included in the final analyses: $414 \mathrm{~TB}$ cases and 438 non-TB participants. The overall median (IQR) age of participants was 38 (31-47) years, with 53\% male. The overall prevalence of HIV-1 was $61.2 \%$ (95\% CI 29.9-36.2\%), and was significantly higher in participants with TB (versus non-TB) and in females (table 1). Compared with those without TB, TB cases were younger, with a lower prevalence of obesity and a greater proportion of men. Of the $414 \mathrm{~TB}$ cases, nine had rifampicin resistance $(2.2 \%)$, all of whom did not have diabetes. Table 1 summarises baseline characteristics of participants, stratified by TB status. 
TABLE 1 Baseline sociodemographic, anthropometric and comorbidity characteristics by tuberculosis (TB) status

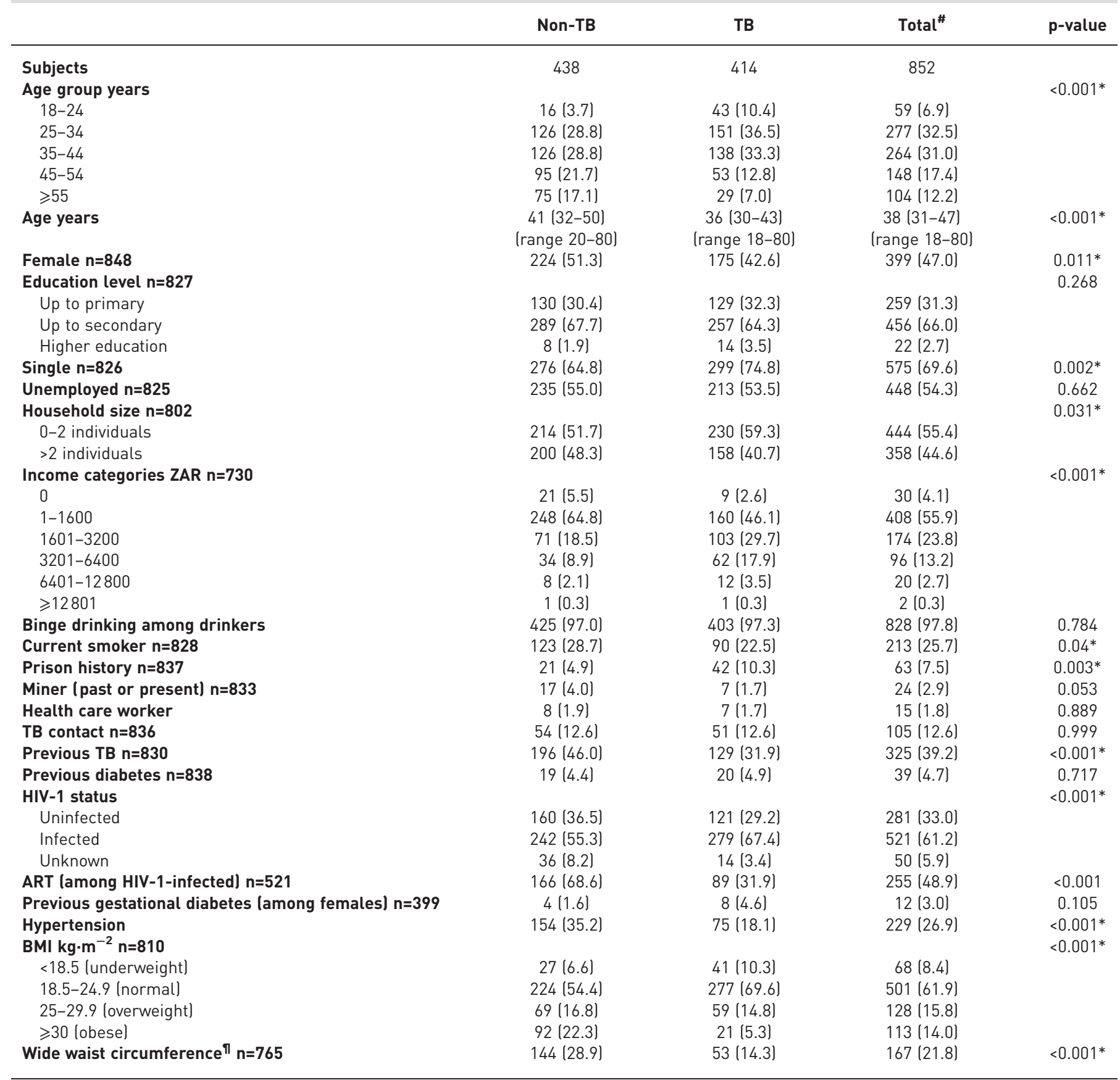

Data are presented as $\mathrm{n}, \mathrm{n}(\%)$ or median (interquartile range), unless otherwise stated. ART: antiretroviral therapy; BMI: body mass index. ${ }^{\#}$ : total $n=852$, unless otherwise stated in column $1 ; \eta^{\eta}: \geqslant 94 \mathrm{~cm}$ males, $\geqslant 88 \mathrm{~cm}$ females. ${ }^{*}: p<0.05$.

Prevalence of diabetes and IGR

The overall prevalence of diabetes (using any of the three diagnostic criteria) was $11.3 \%$ (95\% CI 9.313.6\%); $12.6 \%$ among TB cases (95\% CI 9.7-16.1\%) and 10.1\% (95\% CI 7.6-13.2\%) in non-TB participants $(\mathrm{p}=0.246)$. Among diabetes participants, 59.4\% (57 out of 96) were not previously diagnosed with diabetes $(61.5 \%$ in $\mathrm{TB}$ cases and $56.8 \%$ in non-TB) and all previously diagnosed diabetes patients were on diabetes treatment. Despite treatment, participants with a prior diabetes diagnosis had higher median (IQR) FPG (7.45 (5.2-11.3) versus $\left.5.45(5.1-7.1) \mathrm{mmol} \cdot \mathrm{L}^{-1} ; \mathrm{p}<0.001\right)$ and $\mathrm{HbAlc}(9.7 \%(7.0-$ $11.4 \%$ ) versus $6.5 \%(6.4-6.9 \%) ; \mathrm{p}<0.001)$ levels compared with newly diagnosed diabetes patients. 
The overall statistically significant difference in glycaemic status between participants with and without TB was driven by the prevalence of IGR, which was significantly higher in TB cases than non-TB (65.2\% versus $50.0 \% ; \mathrm{p}<0.001$ ). The prevalence of diabetes and IGR varied by diagnostic test (table 2 ). The majority of diabetes diagnoses were made based on the FPG and HbAlc tests, while IGR was largely driven by a positive $\mathrm{HbAlc}$ test.

The overall prevalence of diabetes was lower in HIV-1-infected patients compared with those uninfected or those with HIV-1 status unknown ( $8.9 \%$ versus $16.0 \%$ or $10.0 \%$, respectively) (table 3 ). While there was no association between TB and diabetes overall, when stratified by HIV-1 status, the prevalence of diabetes was found to be higher in HIV-1-infected TB cases versus HIV-1-infected participants without TB (11.1\% versus 6.2\%; $\mathrm{p}=0.049$ ). This difference was not observed in HIV-1-uninfected participants (table 3 ).

\section{TB/diabetes association and population-attributable risk}

On univariable analysis, age, sex, marital status, household size, income, employment status, previous TB, HIV-1, smoking, hypertension, waist circumference, a history of time in prison, education, BMI and a history of being a miner were all associated with $\mathrm{TB}$ at the $10 \%$ level of significance. These variables were used in turn to build the multivariable model. After adjusting for confounding variables, diabetes was associated with a 2.4 -fold higher odds of TB (95\% CI 1.1-5.2), with this association remaining significant in HIV-1-infected but not HIV-1-uninfected individuals (table 4). Further analysis by diabetes diagnostic test revealed that this significant association was only noted using the HbAlc test. IGR was also associated with a 2.3-fold higher odds of TB (95\% CI 1.6-3.3), with the strongest association shown when the OGTT was used as a diagnostic test compared with other tests (table 4). Unlike the diabetes analysis, the association between TB and IGR (using any test) was significant in both HIV-1-infected and -uninfected individuals, albeit driven by different tests: a significant association using the OGTT in HIV-1-infected individuals and the HbA1c test in HIV-1-uninfected individuals. Based on a $12 \%$ prevalence of diabetes in the general population and the adjusted OR of 2.4, the population-attributable risk of TB due to diabetes is $14 \%$.

\section{Discussion}

The prevalence of diabetes, using any three criteria, was $13 \%$ in $\mathrm{TB}$ cases in Cape Town, largely contributed to by HbA1c. There was also an alarmingly high prevalence of IGR, with $65 \%$ all TB cases having IGR. There is good evidence that TB is associated with transient hyperglycaemia, best measured using FPG and 2-h OGTT (markers of short-term glucose status), unlike HbA1c, which is a marker of glycaemic control over 2-3 months. The finding of the prevalence of diabetes being highest using HbAlc is therefore surprising. One possible explanation is the choice of cut-point. The American Diabetes Association/World Health Organization cut-point is $6.5 \%[30,31]$, but a recent study investigating the diagnostic accuracy of HbAlc against the OGTT in a population survey of black South Africans suggested that the optimal cut-point for detection of diabetes was 6.0\% [35]. However, using this lower cut-point would have resulted in a higher, not lower, prevalence. Similarly, anaemia, common in TB patients, may have resulted in lower HbAlc in TB cases, unless there was iron deficiency with or without anaemia which may result in an increase in HbAlc compared with non-TB participants, without a concomitant rise in glucose indices [36]. It may be that use of HbAlc in the context of TB and/or HIV-1 is flawed, or that the

TABLE 2 Prevalence of diabetes and impaired glucose regulation (IGR), including previously diagnosed diabetes, by diagnostic test

\begin{tabular}{lcccc} 
& Overall & Non-TB & TB & p-value (Chi-squared) \\
\hline Diabetes & & & & \\
Any test & $11.3(9.3-13.6)$ & $10.1(7.6-13.2)$ & $12.6(9.7-16.1)$ & 0.246 \\
FPG & $4.1(3.0-5.7)$ & $3.9(2.4-6.2)$ & $4.4(2.8-6.9)$ & 0.752 \\
OGTT & $3.3(2.3-4.8)$ & $3.5(2.1-5.8)$ & $3.1(1.7-5.3)$ & 0.704 \\
HbA1c & $8.2(6.5-10.2)$ & $6.2(4.3-8.9)$ & $10.2(7.7-13.6)$ & $0.032^{*}$ \\
IGR lexcluding diabetes) & & & & \\
Any test & $57.3(53.7-60.8)$ & $50.0(45.1-54.9)$ & $65.2(60.0-70.0)$ & $<0.001^{*}$ \\
FPG & $10.6(8.6-13.1)$ & $12.5(9.5-16.1)$ & $8.6(6.1-12.2)$ & 0.089 \\
OGTT & $10.6(8.6-13.0)$ & $4.9(3.1-7.5)$ & $16.9(13.3-21.2)$ & $<0.001^{*}$ \\
HbA1c & $39.5(36.1-43.0)$ & $34.1(29.6-38.9)$ & $45.4(40.3-50.6)$ & $0.002^{*}$
\end{tabular}

Data are presented as \% (95\% CI), unless otherwise stated. TB: tuberculosis; FPG: fasting plasma glucose; OGTT: oral glucose tolerance test; HbA1c: glycated haemoglobin. *: $p<0.05$. 


\begin{tabular}{|c|c|c|c|c|}
\hline & Overall & Non-TB & TB & p-value \\
\hline Subjects & 852 & 438 & 414 & \\
\hline HIV-1-uninfected n=281 & $\begin{array}{c}16.0(12.2-20.8) \\
(n=45 / 281)\end{array}$ & $\begin{array}{c}16.9(11.8-23.6) \\
(n=27 / 160)\end{array}$ & $\begin{array}{c}14.9(9.5-22.5) \\
(n=18 / 121)\end{array}$ & 0.651 \\
\hline HIV-1-infected $n=521$ & $\begin{array}{c}8.9(6.7-11.6) \\
(n=46 / 521)\end{array}$ & $\begin{array}{c}6.2(3.8-10.1) \\
(n=15 / 242)\end{array}$ & $\begin{array}{c}11.1(7.9-15.4) \\
(n=31 / 279)\end{array}$ & $0.049 *$ \\
\hline HIV-1 status unknown $n=50$ & $\begin{array}{c}10.0(4.1-22.4) \\
(n=5 / 50)\end{array}$ & $\begin{array}{c}5.6(1.3-20.8) \\
(n=2 / 36)\end{array}$ & $\begin{array}{c}21.4(6.0-54.0) \\
(n=3 / 14)\end{array}$ & 0.093 \\
\hline
\end{tabular}

Data are presented as $\mathrm{n}$ or $\%(95 \% \mathrm{Cl})$, unless otherwise stated. *: $\mathrm{p}<0.05$

cut-point should be revised. Further research is required to better understand factors, such as altered red cell survival, that may influence HbAlc in TB/HIV-1 patients.

We noted a significant association between diabetes and TB, in agreement with the majority of the published literature from other settings, with a 2.4- and 2.3-fold higher odds of TB in diabetes and IGR patients, respectively, and $14 \%$ of $\mathrm{TB}$ cases attributed to diabetes. Only two of these studies were conducted in a setting with a high HIV-1 burden [21, 37] with ORs ranging from 2.2 to 10.7. The coexistence of a high prevalence of HIV-1, TB and diabetes has significant implications for optimal control of each condition, highlighting the importance of targeting TB control interventions, such as intensified TB screening for diabetes and diabetes/HIV-1 patients, to achieve TB elimination goals.

There was also a significant TB/IGR association (adjusted OR 2.3), suggesting that a finding of IGR should also prompt TB screening. While the high prevalence of IGR would support the theory of these cases being transient hyperglycaemia, the finding of the high prevalence strongly driven by HbAlc is a conundrum as it would suggest a more sustained state of hyperglycaemia. Negative outcomes have been associated with TB/diabetes [3]. HIV-1-uninfected TB/diabetes patients are at higher risk of death than TB/HIV patients $[38,39]$, but there is a paucity of research on the association between IGR and TB outcomes to inform the need for interventions to control blood glucose in IGR TB patients.

The association between diabetes and TB also varied depending on the diagnostic test used. We found that the association was largely driven by the HbAlc test, as did BoIllat-Blanco et al. [37] in Tanzania. Using either FPG or OGTT alone as a diabetes diagnostic test did not reveal a statistically significant association. Other studies from Tanzania and Guinea-Bissau also found that IGR/diabetes diagnoses varied when using random blood glucose, FPG and OGTT [19, 21]. Further research is required to adequately define the most appropriate test to identify and monitor diabetes in TB patients, including in HIV-1-co-infected individuals.

On stratification by HIV-1 serostatus, the association between TB and diabetes remained statistically significant only in participants with HIV-1 infection. The reported effect of HIV-1 co-infection on the association between TB and diabetes has been variable. A study using random blood glucose reported reduced odds of diabetes in HIV-1-infected patients [20]. Another study using FPG and OGTT reported similar findings of a stronger TB/diabetes association in HIV-1-uninfected patients [21]. However, a recent study that used all three diabetes diagnostic tests showed a slightly stronger TB/diabetes association among HIV-1-infected individuals, as measured by FPG and OGTT, but not by HbAlc [37]. In our study, the significant TB/diabetes association in HIV-1-infected participants was noted when diabetes was defined using any of the three tests, largely driven by HbAlc. There are limited data on the effect on HIV-1 infection on HbAlc. One study that compared HbAlc values in HIV-1-infected and -uninfected women found slightly lower HbAlc values in HIV-1-infected women after adjustment for fasting glucose values [40]. However, this association was nullified on multivariable analysis, with the differences accounted for by higher red cell mean corpuscular volume in HIV-1-infected individuals. Another study also suggested that HbAlc may underestimate glycaemia in HIV-infected individuals, particularly those with higher mean corpuscular volume, nucleoside reverse transcriptase inhibitor use and lower CD4 count [41].

The association between TB and diabetes was stronger in participants with a prior diabetes diagnosis, with a 3.9-fold higher odds of TB. These participants had a higher median FPG and HbAlc, and thus the stronger association may reflect the greater degree of hyperglycaemia in this group. This is in line with published studies that have shown that poorly controlled diabetes is more strongly associated with TB [42]. Of note, HIV-1-infected patients with a previous diabetes diagnosis were at an even greater risk of TB 
TABLE 4 Univariable and multivariable analysis of the association between tuberculosis and diabetes/impaired glucose regulation (IGR)

\begin{tabular}{|c|c|c|c|c|c|c|c|c|c|c|c|c|}
\hline & \multicolumn{4}{|c|}{ Overall } & \multicolumn{4}{|c|}{ HIV-1-infected } & \multicolumn{4}{|c|}{ HIV-1-uninfected } \\
\hline & $\begin{array}{l}\text { Crude OR } \\
(95 \% \mathrm{CI})\end{array}$ & p-value & $\begin{array}{l}\text { Adjusted } \mathrm{OR}^{\#} \\
\text { (95\% Cl) }\end{array}$ & p-value & $\begin{array}{l}\text { Crude OR } \\
(95 \% \mathrm{CI})\end{array}$ & p-value & $\begin{array}{l}\text { Adjusted OR } \\
\text { (95\% Cl) }\end{array}$ & p-value & $\begin{array}{l}\text { Crude OR } \\
(95 \% \mathrm{CI})\end{array}$ & p-value & $\begin{array}{l}\text { Adjusted } \mathrm{OR}^{\#} \\
\text { (95\% CI) }\end{array}$ & $\mathrm{p}$-value \\
\hline \multicolumn{13}{|l|}{ Diabetes } \\
\hline Overall & $1.3(0.8-2.0)$ & 0.247 & $2.4(1.3-4.3)$ & $0.005^{*}$ & $2.0(1.0-3.8)$ & 0.033 & $2.4(1.1-5.2)$ & $0.030^{*}$ & $1.0(0.5-1.9)$ & 0.995 & $2.4(0.9-6.7)$ & 0.081 \\
\hline $\mathrm{HbA1c}$ & $1.7(1.0-2.9)$ & 0.034 & $2.4(1.2-4.6)$ & $0.012^{*}$ & $2.2(1.1-4.6)$ & 0.032 & $2.4(1.0-5.9)$ & $0.05^{*}$ & $1.5(0.7-3.2)$ & 0.268 & $2.2(0.7-6.4)$ & 0.160 \\
\hline FPG & $1.1(0.6-2.2)$ & 0.725 & $2.3(0.9-5.5)$ & 0.068 & $1.8(0.5-6.0)$ & 0.336 & $2.9(0.7-12.2)$ & 0.148 & $1.1(0.5-2.6)$ & 0.800 & $1.9(0.6-6.4)$ & 0.295 \\
\hline OGTT & $0.9(0.4-1.9)$ & 0.704 & $1.2(0.5-3.3)$ & 0.690 & $1.8(0.5-6.2)$ & 0.322 & $2.5(0.6-10.3)$ & 0.218 & $0.5(0.2-1.7)$ & 0.285 & $0.5(0.1-3.1)$ & 0.491 \\
\hline $\begin{array}{l}\text { Previously diagnosed } \\
\text { diabetes only }\end{array}$ & $1.1(0.6-2.1)$ & 0.717 & $3.7(1.5-9.1)$ & $0.004^{*}$ & $2.1(0.5-8.3)$ & 0.283 & $6.3(1.3-30.8)$ & $0.022^{*}$ & $1.2(0.6-2.6)$ & 0.652 & $3.1(0.9-10.1)$ & 0.066 \\
\hline \multicolumn{13}{|l|}{$\begin{array}{l}\text { IGR (excludes } \\
\text { diabetes cases) }\end{array}$} \\
\hline Overall & $1.9(1.4-2.5)$ & $<0.001$ & $2.3(1.6-3.3)$ & $<0.001 *$ & $2.4(1.7-3.4)$ & $<0.001$ & $2.4(1.5-3.8)$ & $<0.001^{*}$ & $1.2(0.7-2.0)$ & 0.467 & $2.3(1.1-4.7)$ & $0.024^{*}$ \\
\hline $\mathrm{HbA1c}$ & $1.4(1.0-1.8)$ & 0.026 & $1.6(1.1-2.3)$ & $0.009 *$ & $1.6(1.1-2.3)$ & 0.012 & $1.5(1.0-2.3)$ & 0.084 & $1.1(0.7-1.8)$ & 0.569 & $2.2(1.1-4.1)$ & $0.017^{*}$ \\
\hline FPG & $0.8(0.5-1.1)$ & 0.182 & $0.9(0.5-1.5)$ & 0.695 & $1.0(0.6-1.7)$ & 0.867 & $1.2(0.6-2.2)$ & 0.588 & $0.4(0.2-0.8)$ & 0.018 & $0.4(0.1-1.2)$ & 0.116 \\
\hline OGTT & $3.0(1.9-4.8)$ & $<0.001$ & $3.9(2.1-7.0)$ & $<0.001 *$ & $5.0(2.6-9.5)$ & $<0.001$ & $5.4(2.4-12.0)$ & $<0.001^{*}$ & $1.4(0.6-2.9)$ & 0.432 & $2.8(1.0-8.0)$ & 0.058 \\
\hline
\end{tabular}

HbA1c: glycated haemoglobin; FPG: fasting plasma glucose; OGTT: oral glucose tolerance test. " : adjusted for sex, age, household size, income, hypertension (baseline), previous miner, previous prisoner, marital status, work status and HIV-1 status. *: $p<0.05$. 
with the adjusted OR increasing from 2.4 to 6.3 . In this and other studies $[13,20]$, a high percentage of diabetes among TB patients was previously undiagnosed. Data from chronic disease audits across more than 100 primary care facilities in the Western Cape Province of South Africa, within which Cape Town is located, have reported that $<15 \%$ of diabetes patients attending clinics have HbAlc levels $<7 \%$ (Western Cape Dept of Health, Cape Town, South Africa; unpublished data). Our results therefore strongly support the need to prioritise improved diabetes management as a TB control interventions.

\section{Strengths and limitations}

A significant strength of this study was the performance of three different tests for diabetes using venous blood samples with all tests performed in an accredited national laboratory. In addition, multivariable analysis of the association between TB and diabetes/IGR adjusted for the most prevalent and significant known confounders, including a previous history of time in prison, mine work and household income, as well as HIV-1 infection.

Our study had a number of limitations. Glycaemia was measured at baseline and it is possible that the hyperglycaemia was transient. We attempted to limit the effect of transient hyperglycaemia on the reported $\mathrm{TB} /$ diabetes association by selecting non-TB participants from patients with respiratory symptoms that later resolved. Studies have shown an association between hyperglycaemia at TB diagnosis (even when transient) and TB treatment failure and death [37], highlighting the clinical importance of intervening in patients with baseline hyperglycaemia to improve TB outcomes. Small numbers after stratification by HIV-1 status meant we were unable to investigate the effect of ART or CD4 count. In addition, given that the association between TB and diabetes was significant overall, and that the study was powered to detect this association, the lack of statistical significance noted in HIV-1-uninfected participants may be due to a reduced statistical power after stratification; this study is not able to exclude the possibility of an association in HIV-uninfected individuals. Finally, given the association between diabetes and poorer TB outcomes, the exclusion of critically ill patients due to their inability to give consent may have introduced selection bias with possible exclusion of TB patients with diabetes. Similarly, while the multidrug-resistant (MDR)-TB prevalence found in this study is similar to the national prevalence $(2.8 \%)$ [43], given the poor outcomes associated with MDR-TB, the exclusion of critically ill patients may also have resulted in a greater proportion of excluded patients with MDR-TB.

\section{Conclusion}

Diabetes is a significant contributor to the TB burden in this high TB/HIV-1/diabetes burden setting. Our study utilised international guideline-approved diabetes tests and found a high prevalence of diabetes in $\mathrm{TB}$ cases, and a significant association between $\mathrm{TB}$ and diabetes, even in the context of HIV-1 co-infection, with greater odds of TB in participants who were previously diagnosed with diabetes. These findings point to the importance of screening for diabetes in those with TB and those with HIV-1, who are already at high risk for developing TB. A surprisingly high prevalence of IGR was also found in this study, emphasising the need for further investigation into the TB outcomes and management of this group. The interpretation of our findings is complex because of the variations in diabetes prevalence and TB/diabetes association by different diagnostic tests. Existing glycaemia measures can be affected by HIV-1, ART, anaemia, acute infection, red cell survival and iron status. This highlights the need for more accurate markers of glycaemia that are independent of these factors in order to inform policy on how best to screen for diabetes at the time of TB diagnosis in (often low-resource and primary care) settings with a high burden of HIV-1 and TB.

\section{Acknowledgements}

Author contributions: T. Oni designed the study and was principal investigator. T. Oni, N.S. Levitt and R.J. Wilkinson were involved in the design of the study. N. Berkowitz led participant recruitment, retention, phenotypic characterisation of all participants and data management. R. Goliath led study coordination, and contributed to patient recruitment, follow-up and collection of data. Data analysis and interpretation were conducted by M. Kubjane and T. Oni. All authors were involved in the drafting of the manuscript, led by T. Oni. The final manuscript draft was approved by all authors. T. Oni had full access to all the data in the study and takes responsibility for the integrity of the data and the accuracy of the data analysis.

\section{References}

1 Patra J, Jha P, Rehm J, et al. Tobacco smoking, alcohol drinking, diabetes, low body mass index and the risk of self-reported symptoms of active tuberculosis: individual participant data (IPD) meta-analyses of 72,684 individuals in 14 high tuberculosis burden countries. PLoS One 2014; 9: e96433.

2 Jeon CY, Murray MB. Diabetes mellitus increases the risk of active tuberculosis: a systematic review of 13 observational studies. PLoS Med 2008; 5: e152.

3 Baker MA, Harries AD, Jeon CY, et al. The impact of diabetes on tuberculosis treatment outcomes: a systematic review. BMC Med 2011; 9: 81. 
4 Magee MJ, Kempker RR, Kipiani M, et al. Diabetes mellitus is associated with cavities, smear grade, and multidrug resistant tuberculosis in Georgia. Int J Tuberc Lung Dis 2015; 19: 37-54.

5 GBD 2013 Mortality and Causes of Death Collaborators. Global, regional, and national age-sex specific all-cause and cause-specific mortality for 240 causes of death, 1990-2013: a systematic analysis for the Global Burden of Disease Study 2013. Lancet 2015; 385: 117-171.

6 Raghuraman S, Vasudevan KP, Govindarajan S, et al. Prevalence of diabetes mellitus among tuberculosis patients in urban Puducherry. N Am J Med Sci 2014; 6: 30-34.

7 Padmalatha P, Hema K. Study on prevalence of diabetes mellitus in tuberculosis patients attending a tertiary care hospital in Guntur, Andhra Pradesh. Indian J Basic Appl Med Res 2014; 4: 494-498.

8 Balakrishnan S, Vijayan S, Nair S, et al. High diabetes prevalence among tuberculosis cases in Kerala, India. PLoS One 2012; 7: e46502.

9 Viswanathan V, Kumpatla S, Aravindalochanan V, et al. Prevalence of diabetes and pre-diabetes and associated risk factors among tuberculosis patients in India. PLoS One 2012; 7: 1-9.

10 Gupta S, Shenoy VP, Bairy I, et al. Diabetes mellitus and HIV as co-morbidities in tuberculosis patients of rural South India. J Infect Public Health 2011; 4: 140-144.

11 Restrepo BI, Camerlin AJ, Rahbar MH, et al. Cross-sectional assessment reveals high diabetes prevalence among newly-diagnosed tuberculosis cases. Bull World Health Organ 2011; 89: 352-359.

12 Alladin B, Mack S, Singh A, et al. Tuberculosis and diabetes in Guyana. Int J Infect Dis 2011; 15: e818-e821.

13 Olayinka AO, Anthonia O, Yetunde K. Prevalence of diabetes mellitus in persons with tuberculosis in a tertiary health centre in Lagos, Nigeria. Indian J Endocrinol Metab 2013; 17: 486-489.

14 Faurholt-Jepsen D, Range N, PrayGod G, et al. The role of anthropometric and other predictors for diabetes among urban Tanzanians with tuberculosis. Int J Tuberc Lung Dis 2012; 16: 1680-1685.

15 Baldé NM, Camara A, Camara LM, et al. Associated tuberculosis and diabetes in Conakry, Guinea: Prevalence and clinical characteristics. Int J Tuberc Lung Dis 2006; 10: 1036-1040.

16 Marais RM. Diabetes mellitus in black and coloured tuberculosis patients. S Afr Med J 1980; 57: 483-484.

17 International Diabetes Federation. Diabetes Atlas. 7th Edn. www.diabetesatlas.org Date last accessed: April 18, 2017.

18 Peer N, Steyn K, Lombard C, et al. Rising diabetes prevalence among urban-dwelling black South Africans. PLoS One 2012; 7: e43336.

19 Haraldsdottir TL, Rudolf F, Bjerregaard-Andersen MC, et al. Diabetes mellitus prevalence in tuberculosis patients and the background population in Guinea-Bissau: a disease burden study from the capital Bissau. Trans $R$ Soc Trop Med Hyg 2015; 109: 400-407.

20 Kibirige D, Ssekitoleko R, Mutebi E, et al. Overt diabetes mellitus among newly diagnosed Ugandan tuberculosis patients: a cross sectional study. BMC Infect Dis 2013; 13: 122

21 Faurholt-Jepsen D, Range N, PrayGod G, et al. Diabetes is a risk factor for pulmonary tuberculosis: a case-control study from Mwanza, Tanzania. PLoS One 2011; 6: e24215.

22 Bailey SL, Ayles H. Association between diabetes mellitus and active tuberculosis in Africa and the effect of HIV. Trop Med Int Health 2017; 22: 261-268.

23 Evans D. Ten years on ART - where to now? S Afr Med J 2013; 103: 229-231.

24 TBfacts.org. TB statistics - global, regional, age \& high burden. www.tbfacts.org/tb-statistics Date last accessed December 20, 2016

25 Levitt NS, Peer N, Steyn K, et al. Increased risk of dysglycaemia in South Africans with HIV; especially those on protease inhibitors. Diabetes Res Clin Pract 2016; 119: 41-47.

26 Karamchand S, Leisegang R, Schomaker M, et al. Risk factors for incident diabetes in a cohort taking first-line nonnucleoside reverse transcriptase inhibitor-based antiretroviral therapy. Medicine 2016; 95: e2844.

27 Statistics South Africa. Mortality and Causes of Death in South Africa, 2013: Findings from Death Notification. www.statssa.gov.za/publications/P03093/P030932013.pdf Date last accessed: December 20, 2016.

28 World Health Organization. Global Tuberculosis Report 2014. http://apps.who.int/iris/bitstream/10665/137094/1/ 9789241564809_eng.pdf Date last accessed: December 20, 2016.

29 Department of Health, Republic of South Africa. National Tuberculosis Management Guidelines 2014. www.nicd. ac.za/assets/files/Acrobat\%20Document2.pdf Date last accessed: December 20, 2016.

30 World Health Organization. Definition and Diagnosis of Diabetes Mellitus and Intermediate Hyperglycemia. 2006. www.who.int/diabetes/publications/diagnosis_diabetes2006/en/index.html Date last accessed: February 3, 2015.

31 American Diabetes Association. Standards of Medical Care in Diabetes - 2013. Diabetes Care 2013; 36: Suppl. 1, S11-S66.

32 Alberti KGMM, Zimmet P, Shaw J. Metabolic syndrome - a new world-wide definition. A consensus statement from the International Diabetes Federation. Diabet Med 2006; 23: 469-480.

33 World Health Organization. STEPS Instrument for Chronic Disease Risk Factor Surveillance 2009. www.who.int/ chp/steps/STEPS_Instrument_v2.1.pdf Date last accessed: December 20, 2016.

34 Greenland S, Robins JM. Conceptual problems in the definition and interpretation of attributable fractions. Am J Epidemiol 1988; 128: 1185-1197.

35 Hird TR, Pirie FJ, Esterhuizen TM, et al. Burden of diabetes and first evidence for the utility of HbAlc for diagnosis and detection of diabetes in urban black South Africans: the Durban Diabetes Study. PLoS One 2016; 11: e0161966.

36 English E, Idris I, Smith G, et al. The effect of anaemia and abnormalities of erythrocyte indices on HbAlc analysis: a systematic review. Diabetologia 2015; 58: 1409-1421.

37 Boillat-Blanco N, Ramaiya KL, Mganga M, et al. Transient hyperglycemia in patients with tuberculosis in Tanzania: Implications for diabetes screening algorithms. J Infect Dis 2016; 213: 1163-1172.

38 Faurholt-Jepsen D, Range N, Praygod G, et al. Diabetes is a strong predictor of mortality during tuberculosis treatment: a prospective cohort study among tuberculosis patients from Mwanza, Tanzania. Trop Med Int Heal 2013; 18: 822-829.

39 Workneh MH, Bjune GA, Yimer SA. Diabetes mellitus is associated with increased mortality during tuberculosis treatment: a prospective cohort study among tuberculosis patients in South-Eastern Amahra Region, Ethiopia. Infect Dis Poverty 2016; 5: 22. 
40 Glesby MJ, Hoover DR, Shi Q, et al. Glycated haemoglobin in diabetic women with and without HIV infection: data from the Women's Interagency HIV Study. Antivir Ther 2010; 15: 571-577.

41 Monroe AK, Glesby MJ, Brown TT. Diagnosing and managing diabetes in HIV-infected patients: current concepts. Clin Infect Dis 2015; 60: 453-462.

42 Yoon YS, Jung J-W, Jeon EJ, et al. The effect of diabetes control status on treatment response in pulmonary tuberculosis: a prospective study. Thorax 2016; 72: 263-270.

43 National Institute for Communicable Diseases. South African Tuberculosis Drug Resistance Survey 2012-14. www. nicd.ac.za/assets/files/K-12750\%20NICD\%20National\%20Survey\%20Report_Dev_V11-LR.pdf Date last accessed: March 31, 2017. 